\title{
РЕГУЛЯТОРНЫЕ ХАРАКТЕРИСТИКИ \\ СУБЪЕКТИВНОГО ОБРАЗА СОСТОЯНИЯ В ТРУДНОЙ ЖИЗНЕННОЙ СИТУАЦИИ
}

\begin{abstract}
Аннотация. Статья раскрывает специфику регуляторной активности субъекта трудной жизненной ситуации в зависимости от выраженности характеристик образа состояния в ней. Целью представленного эмпирического исследования был анализ формальных параметров образа состояния и его связь с общей регуляторной активностью. Проводилось сравнение мощности, структурированности и интенсивности образа в различных условиях воспроизведения трудной ситуации. Изучалась дифрференцированная роль параметров образа в актуально действующей ситуации, при воспроизведении положительного опыта решения проблем и в условиях несовладания с трудностями. Исследование базируется на общеметодологических положениях концепции стиля осознанной саморегуляции произвольной активности субъекта В.И. Моросановой. Показана динамика субъективных характеристик при снижении адаптированности к трудной ситуации и влияние изменений в образе состояния на общий уровень саморегуляции. Доказано, что образ состояния дифреренцировано влияет на регуляторную активность. Его мощность более всего детерминирует процессы управления ситуаций и своей активностью в ней. Полученные эмпирические данные поднимают актуальный вопрос о возможности и необходимости разработки технического обеспечения коррекционной помощи субъекту трудной жизненной ситуации с учётом механизмов воздействия на мощность, интенсивность и структурированность образа психического состояния.

Ключевые слова: образ состояния, произвольная активность, осознанная саморегулячия, мощность, структурированность, интенсивность, трудная жизненная ситуация, субъект ситуации, адаптированность, совладание с трудностями.

Review. The article reveals the specifics of the regulatory activity of an actor in difficult situations depending on the intensity of the features of mental state images. The objective of the empirical study was to analyze the formal parameters of mental state image and its relation to the overall regulatory activity. The author of the article has carried out the comparison of the power, structure and intensity of images appearing under various conditions. The author has also studied the differentiated role of the image parameters in an actual situation when reproducing positive experience of problem solution as well as conditions when difficulties are not overcome. The study is based on the provisions of the general methodological concept of conscious self-regulation of voluntary activity as described by V. Morosanova. The author describes the dynamics of subjective features when the level of adaptation to a difficult situation is low as well as the influence of changes in the state image on the overall level of self-regulation. The author proves that the state image has a differentiated influence on regulatory activity. The intensity of the state image determines the processes of situation management as well as the actor's activity in such a situation. These empirical data raises an urgent question regarding the possibility and the need for technical assistance to provide assistance to an individual experiencing a difficult life situation taking into account mechanisms of influence on power, intensity and structure of the image of mental state.

Key words: coping, adaptation, actor in a situation, difficult life situations, image of mental state, self-conscious regulation, voluntary activity, power, structured, intensity.
\end{abstract}

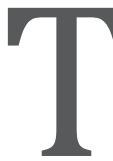

рудная жизненная ситуация как система противоречий, требующая для разрешения приложения целенаправленной активности, отражается в представлении посредством опыта переживания. Фиксация в сознании конкретного переживания формирует образ того состояния, через которое субъект выражает отношение к себе в сложных условиях. Мы определяем субъективный образ психического состояния вслед за А.О. Прохоровым как «...семанти- 


\section{Ключи творчества}

ческое пространство, включающее в себя следы переживаний ранее осуществленных деятельностей, поведения, реакций и др. Это следы «сцепления» семантического пространства с ситуациями и обстоятельствами жизнедеятельности субъекта» [1, c. 544]. Содержание образа состояния обусловлено работой памяти, но в то же самое время, он актуален и направляет активность «здесь-и-теперь» [2]. Кроме того, воссоздавая прошлые впечатления, образ антиципирует и переориентирует своё содержание в будущее [3; 4], а это, в свою очередь, направляет и регулирует активность в схожей ситуации, придавая ей заведомо трудный характер и повышая субъективную значимость [5, с. 28].

Поскольку основной характеристикой субъекта выступает активность, способная изменять ситуацию и себя, решающая роль в преодолении жизненных трудностей принадлежит регуляторной деятельности. В частности, осознанная саморегуляция, соотносимая с системой представлений о себе, в конкретной ситуации отвечает за процессы принятия цели, контроля побуждений и может выступать условием их коррекции [6].

Наше обращение к проблеме субъективного образа состояния обусловлено важностью нахождения способов оказания психологической помощи людям, оказавшимся в сложных условиях жизни. Если образ состояния подвижен, гибок, имеет дифференцированные формальные показатели, а его генезис связан с процессами осознания и рефлексии [7], то воздействуя на него можно вносить изменения и в сам процесс регуляции произвольной ситуативной активности.

При проведении исследования нас интересовало, во-первых, динамика формальных показателей образа состояния при повышении напряжения трудной ситуации; во-вторых, связь образа состояния с регуляторной активностью в трудных условиях жизни.

\section{Организационно-методическое обеспечение исследования}

Экспериментальную выборку составили субъекты трудной жизненной ситуации с различной степенью адаптированности к ней (224 чел.). При подборе экспериментальных групп для нас важно было определить те психофизиологические и эмоциональные характеристики, которые возникают вследствие успешного или неуспешного регулирования в ситуации своих потребностей, притяза- ний, эмоций и др. Для обозначения этих состояний чаще всего и используют понятие адаптированности, понимая под ним степень результативности и оптимальности приспособительных механизмов в конкретных условиях. Диагностический комплекс включал психобиографические методы, контентанализ отчётов о самочувствии и психометрические шкалы оценки состояний. Данные систематизировались иерархическим кластерным анализом с агломеративным алгоритмом. В результате было выделено три кластера (и аналогичные экспериментальные группы) соответственно типам ситуаций, каждая из которых представлена испытуемыми с различными параметрами оценки её трудности и уровнями адаптированности к ней.

Первый кластер - ситуация слабой напряжённости - включает 76 испытуемых с оптимальной адаптацией к трудным условиям. Такие ситуации продолжаются 1-3 недели и разрешаются собственными усилиями. Всех представителей первой группы характеризует эмоциональная стабильность, положительная самооценка, устойчивый жизненный интерес, целеустремленность, решительность и способность достигать поставленных целей. Эпизодически возникающий психологический дискомфорт не нарушает адаптационного потенциала.

Второй кластер - интенсивно трудная ситуация - представлен 74 испытуемыми. Интенсивно трудная ситуация имеет затяжной характер (достигает 2-3 месяцев), и обусловливает приложение дополнительных ресурсов для совладания с ней. Испытуемые второго кластера отличаются снижением продуктивности и работоспособности, неудовлетворённой реализацией жизненных планов, зависимостью от конкретных потребностей. Вместе с тем, у них поддерживается жизненный интерес, а в ряде случаев увеличивается активность в достижении целей.

Третий кластер - чрезмерно трудная ситуация, вызывающая у 74 испытуемых напряжённую адаптацию. Представителям третьей группы свойственно пассивное принятие жизненных неудач, эмоциональная лабильность, подвижная самооценка, снижение активности, апатичность, раздражительность, общая ослабленность и истощаемость. Чрезмерно трудные ситуации продолжаются более 3 месяцев и зачастую распространяются на иные жизненные сферы.

Механизмы саморегуляции произвольной активности субъекта в трудной жизненной си- 
туации изучались с помощью опросника «Стиль саморегуляции поведения» В.И. Моросановой [8]. Опросник диагностирует развитость индивидуального профиля саморегуляции, включающего показатели планирования, моделирования, программирования, оценки результатов, а также показатели развития таких регуляторных качеств, как гибкость и самостоятельность. Для корреляционного и регрессионного анализов мы использовали только данные общего регуляторного уровня испытуемых.

Формальные параметры субъективного образа ситуации выделялись и анализировались с помощью специально разработанной нами для этой цели методики. На первом этапе испытуемым необходимо было вспомнить и назвать все свои характеристики последовательно по трём условиям:

1 - актуально действующая трудная жизненная ситуация;

2 - бывшая в опыте трудная жизненная ситуация, близкая по интенсивности и содержанию к актуальной, которую испытуемый смог успешно самостоятельно разрешить. Субъективной оценкой успешного разрешения выступало чувство удовлетворения от полученного в ситуации результата;

3 - бывшая в опыте трудная жизненная ситуация, близкая по интенсивности и содержанию к актуальной, которую испытуемый не смог успешно разрешить или разрешил неуспешно. Субъективная оценка неуспешного разрешения - чувство неудовлетворения от достигнутого результата по разрешению трудности.

Проведя первую опытную серию, мы заметили негативную тенденцию: испытуемые затруднялись самостоятельно называть психические качества или делали это крайне неуверенно. Поэтому в дальнейшем им были предложены 5 ориентиров (эмоции, познавательная деятельность, личностные свойства, поведение, психофизиологическая активность) с интерпретацией и толкованием каждого пункта. Списки воспроизведенных испытуемыми свойств корректировались для того, чтобы убрать дублирующие и невалидные характеристики.

На втором этапе нужно было оценить каждое свойство из трёх скорректированных списков по пятибалльной шкале.

Эмпирические данные позволили нам подсчитать индексы мощности, структурированности и интенсивности, а также вывести индексы расхождения между образами состояния в различных ситуациях.
Индекс мощности - количество свойств, которые субъект приписывает себе как автору и исполнителю трудной жизненной ситуации. Образ тем мощнее, чем более развёрнуто его содержание, при этом равнозначность распределения по параметрам оценки и вектору свойства (позитивной негативный) может игнорироваться.

Индекс структурированности подсчитывался после введения для испытуемых ориентиров, выступающих элементами структуры: эмоции, познавательная деятельность, личностная активность, поведение, психофизиологическая активность. Структурированность отражает наличие логичных связей между отдельными элементами образа. Мы полагаем, что оформленная структура образа стремится к целостности, которая условно его замыкает и таким образом управляет всей активностью субъекта в трудной ситуации. Испытуемый получал по 1 баллу за наличие каждого из пяти элементов. Наличием считалось приписывание себе хотя бы 2 характеристик в элементе; меньшее количество оценивалось в 0 баллов. После этого подсчитывалось соотношение (равновесность) характеристик внутри подструктур. Если соотносились пять элементов с примерно равнозначным количеством характеристик, испытуемый получал 4 балла, если четыре элемента 3 балла, если три - 2 балла, равновесность двух элементов оценивалась в 1 балл и, если структуры не соотносились в количественном выражении, испытуемый получал 0 баллов.

Индекс интенсивности - параметр силы образа, выраженный в баллах. Эта характеристика обозначает меру, степень напряжённости, насыщенности образа. Посчитывался путём сложения оценок испытуемыми своих качеств.

Для определения степени согласования рассогласования параметров в различных экспериментальных условиях мы ввели индекс расхождения, который определялся на основе математической операции разности множеств. Индекс расхождения показывает постоянство или изменчивость мощности, структуры и силы образа состояния в зависимости от воспроизведения испытуемыми актуального и прошлого опыта. Мы полагаем, с одной стороны, что именно изменчивость образа детерминирует регуляцию активности субъекта в ситуации, а, с другой, что подобная вариативность может выступать содержанием коррекционного воздействия на регуляторные процессы. 
ИРоус - индекс расхождения между образом актуального состояния и образом состояния при успешном совладании - количественный показатель рассогласования приписываемых субъектом характеристик актуального состояния в трудной жизненной ситуации и характеристик состояния при успешном совладании с предыдущей ситуацией.

ИРонус - индекс расхождения между образом актуального состояния и образом состояния при неуспешном совладании - количественный показатель рассогласования приписываемых субъектом характеристик актуального состояния в трудной жизненной ситуации и характеристик состояния при ранее неуспешном опыте совладания с проблемами.

Достоверность расхождения образов по трём экспериментальным условиям проверялась критерием знаков (G - критерием).

\section{Результаты исследования}

В таблице 1 представлены средние значения формальных параметров образа состояния испытуемых различных кластеров.

Параметр «мощность» наиболее выражен в первой группе, где количество приписываемых себе характеристик мало различаются по трём условиям. Во второй и третьей группах индексы мощности образов постепенно снижаются, различия между образами в ситуационных условиях становятся заметнее. Предположительно, образ тем мощнее, чем успешнее адаптация субъекта к трудным обстоятельствам. Индекс расхождения мощности образа состояния при успешном разрешении трудностей (как ресурсный показатель) менее всего выражен в первой группе; его показатели во втором и третьем кластерах демонстрируют постепенное статистически значимое повышение, а, с учётом достоверности сдвига, и действенную эффективность. Вместе с тем, все значения индекса находятся в диапазоне 1-2, что свидетельствует о небольшой ресурсной зоне мощности образов. Индекс расхождения мощности образа при неуспешном совладании имеет равнозначные значения в первой и второй группах и существенно повышается в третьей. Модальность сдвига показывает, что в первой группе характеристики образа снижаются, а во второй и третьей - повышаются, но повышаются за счёт приписывания себе непродуктивных качеств. Получается, что чем ниже адаптация к трудным условиям, тем выше вариативность неуспешных характеристик. Наиболее ярко это про-

Средние значения формальных показателей образа состояния

Таблица 1 у субъектов трудной жизненной ситуации

\begin{tabular}{|c|c|c|c|c|c|c|c|}
\hline \multirow{2}{*}{ Кластеры } & \multicolumn{7}{|c|}{ Средние значения индексов } \\
\hline & AOC & OyC & OHУC & ИРоус & Goyc & ИРонус & Goнус \\
\hline \multicolumn{8}{|c|}{ Мощность } \\
\hline Ситуация слабой напряжённости & 23.72 & 23.40 & 23.08 & $1.61(-)$ & 31 & $1.56(-)$ & $22 * *$ \\
\hline Интенсивно трудная ситуация & 20.46 & 19.11 & 21.68 & $1.96(-)$ & $10^{*}$ & $1.57(+)$ & $10^{*}$ \\
\hline Чрезмерно трудная ситуация & 19.01 & 17.54 & 22.61 & $2.09(-)$ & $15^{*}$ & $4.20(+)$ & $5^{*}$ \\
\hline \multicolumn{8}{|c|}{ Структурированность } \\
\hline Ситуация слабой напряжённости & 7.53 & 7.68 & 7.49 & $0.62(+)$ & 15 & $0.76(-)$ & 22 \\
\hline Интенсивно трудная ситуация & 6.53 & 6.18 & 5.61 & $0.84(-)$ & $17 *$ & $1.18(-)$ & $10^{*}$ \\
\hline Чрезмерно трудная ситуация & 5.74 & 5.77 & 5.69 & $0.86(+)$ & 29 & $0.88(-)$ & 26 \\
\hline \multicolumn{8}{|c|}{ Интенсивность } \\
\hline Ситуация слабой напряжённости & 69.95 & 70.10 & 70.59 & 1.97 & 34 & 2.16 & 30 \\
\hline Интенсивно трудная ситуация & 61.68 & 52.54 & 66.27 & $9.23(-)$ & 6* & $5.64(+)$ & 9* \\
\hline Чрезмерно трудная ситуация & 58.89 & 51.09 & 66.20 & $9.11(-)$ & $8^{*}$ & $6.74(+)$ & 9* \\
\hline
\end{tabular}

\section{Примечания.}

* Сдвиг достоверен при Р < 0.01;

** сдвиг достоверен при $\mathrm{P}<0.05$.

АОС - актуальный образ состояния;

ОУС - воспроизведение образа состояния при успешном совладании с предыдущей трудной жизненной ситуацией;

ОНУС - воспроизведение образа состояния при несовладании или неуспешном совладании с предыдущей трудной жизненной ситуацией;

ИРоус - индекс расхождения между образом актуального состояния и образом состояния при успешном совладании;

ИРонус - индекс расхождения между образом актуального состояния и образом состояния при неуспешном совладании;

$\mathrm{G}_{\text {оус }}$ - критерий знаков ИРоус;

$\mathrm{G}_{\text {онус }}-$ критерий знаков ИРонус. 
является при напряжённой адаптации в третьей группе.

Показатели «структурированности» комплексно отражаются в группе испытуемых слабо напряжённой ситуации: разброс средних значений минимальный, а количество выделенных элементов наивысшее по всей выборке. В остальных группах структурированность каждого образа снижается по мере придания большей трудности жизненной ситуации. Индекс расхождения структурированности образа при успешном совладании не значимо выражен в первой и третьих группах, достоверным можно признать сдвиг только у испытуемых второго кластера. Возможно, рассогласование между структурами образа состояния при актуальном отражении трудной жизненной ситуации и при воспроизведении предыдущего успешного опыта соотносится с приложением дополнительных ресурсов для адаптации к сложным условиям. Аналогично распределены значения индекса расхождения структурированности при неуспешном совладании, за тем исключением, что во второй группе его показатели превышают единицу и являются наиболее уверенным параметром рассогласования по всей выборке. Модальность сдвига подтверждает снижение структурированности в этой группе.

«Интенсивность» - самая изменчивая характеристика субъективного образа состояния. В первой группе интенсивность образов по трём экспериментальным условиям дифференцируется несущественно, во второй и третьей - различия становятся заметнее. В этих группах обращает на себя внимание факт превышения значений интенсивности образа состояния при неуспешном совладании над другими. Индекс расхождения интенсивности образа при успешном совладании в первой группе показывает незначительную вариативность двух условий (в 1-2 балла), во второй и третьей группах он становится статистически значимым и достигает 9 баллов. Отрицательная модальность сдвига доказывает снижение у испытуемых силы действия субъективного образа при восприятии успешного предыдущего опыта. Положительный опыт самоотношения, таким образом, не переносится на действующую в настоящее время ситуацию. Индекс расхождения интенсивности образа при неуспешном совладании в группе испытуемых ситуации слабой напряжённости выражен 2 баллами, в отличие от второго и третьего кластера (5-7 баллов). В этих группах отрицательный сдвиг свидетельствует о том, что при повышении трудности ситуации образ себя как некомпетентного в решении проблем становится сильнее.

Таким образом, все формальные параметры образов состояния испытуемых меняются в зависимости от снижения степени их адаптированности к трудной жизненной ситуации. Наиболее динамичным оказывается сила образа, выраженная в субъективной количественной оценке своих характеристик.

Если субъективный образ мобилен, связан с восприятием сложности жизненных обстоятельств, то могут ли его изменения сопровождаться изменениями уровня саморегуляции произвольной активности? Данные корреляционного анализа связи образа состояния с регуляторной активностью представлены в таблице 2.

Корреляционный анализ проводился по каждой группе испытуемых. Мощность образов имеет значимые корреляционные связи с саморегуляцией во второй и третьей группах. В интенсивно и чрезмерно трудных жизненных обстоятельствах уровень осознанной саморегуляции тем выше, чем больше мощность образов по всем трём условиям. Степень рассогласования мощности образов не коррелирует с регуляторной активностью. Структурированность положительно связана с общим регуляторным уровнем только у испытуемых второго кластера и только в отношении актуально действующей ситуации. Другими словами, количество связей внутри образов не определяет регуляторной активности у субъектов трудной жизненной ситуации. Интенсивность прямо коррелирует между всеми образами и саморегуляцией во второй и третьей группах, при этом индекс рассогласования образа неуспешного совладания имеет прямую связь с саморегуляцией у испытуемых чрезмерно трудной ситуации.

Если рассматривать отдельно группы, то видно, что у испытуемых с привычным способом совладания (ситуация слабой напряжённости) осознанная саморегуляция не связана с формальными показателями образа состояния. Предположительно, здесь менее всего регуляторная динамика объясняется изменениями в системе представлений о себе в трудных жизненных условиях. При повышении трудности ситуации уровень саморегуляции становится тем выше, чем мощнее и сильнее выражены образы актуального состояния, образы состояния при успешном и неуспешном разрешении трудностей. 
Ключи творчества

Таблица 2

Данные связи образа состояния и уровня саморегуляции у субъектов трудной жизненной ситуации

\begin{tabular}{|c|c|c|c|c|c|}
\hline \multirow{2}{*}{ Кластеры } & \multicolumn{5}{|c|}{ Коэффициенты корреляции (по Ч. Спирмену) } \\
\hline & AOC & OУC & ОНУс & ИРоyс & ИРонус \\
\hline \multicolumn{6}{|c|}{ Мощность } \\
\hline Ситуация слабой напряжённости & -0.087 & -0.067 & -0.107 & 0.078 & -0.025 \\
\hline Интенсивно трудная ситуация & 0.671 & 0.578 & 0.536 & -0.205 & -0.206 \\
\hline Чрезмерно трудная ситуация & 0.671 & 0.505 & 0.444 & -0.185 & -0.024 \\
\hline \multicolumn{6}{|c|}{ Структурированность } \\
\hline Ситуация слабой напряжённости & -0.053 & -0.009 & -0.045 & 0.012 & 0.017 \\
\hline Интенсивно трудная ситуация & 0.551 & 0.258 & 0.156 & -0.290 & -0.374 \\
\hline Чрезмерно трудная ситуация & 0.351 & 0.315 & 0.274 & -0.046 & -0.071 \\
\hline \multicolumn{6}{|c|}{ Интенсивность } \\
\hline Ситуация слабой напряжённости & 0.034 & 0.056 & 0.034 & 0.010 & 0.009 \\
\hline Интенсивно трудная ситуация & 0.603 & 0.582 & 0.690 & -0.035 & 0.079 \\
\hline Чрезмерно трудная ситуация & 0.623 & 0.546 & 0.678 & -0.088 & 0.423 \\
\hline
\end{tabular}

Влияние образа состояния на уровень осознанной саморегуляции

таблица 3 (результаты регрессионного анализа)

\begin{tabular}{|c|c|c|c|}
\hline Предикторы & $r$ & $\beta$ & $d^{2}$ \\
\hline \multicolumn{4}{|c|}{ Мощность } \\
\hline $\mathrm{AOC}$ & 0.563 & 0.586 & 0.33 \\
\hline OYC & 0.556 & 0.413 & 0.23 \\
\hline OHYC & -0.02 & -0.296 & 0.005 \\
\hline Статистика модели & & $=0.748$ & \\
\hline \multicolumn{4}{|c|}{ Структурированность } \\
\hline $\mathrm{AOC}$ & 0.594 & 0.465 & 0.28 \\
\hline OYC & 0.411 & 0.136 & 0.06 \\
\hline OHYC & 0.482 & 0.187 & 0.09 \\
\hline \multirow{2}{*}{\multicolumn{4}{|c|}{$\frac{\mathrm{R}=0.659 ; \mathrm{R}^{2}=0.434 ; \mathrm{F}=25.42}{\text { Интенсивность }}$}} \\
\hline & & & \\
\hline $\mathrm{AOC}$ & 0.353 & -0.006 & -0.002 \\
\hline OYC & 0.657 & 0.735 & 0.482 \\
\hline OHYC & 0.271 & -0.387 & -0.104 \\
\hline Статистика модели & \multicolumn{3}{|c|}{$R=0.613 ; R^{2}=0.376 ; F=13.42$} \\
\hline
\end{tabular}

Для проверки гипотезы о том, что мощность, структурированность и интенсивность образа состояния субъекта выступают предикторами его регуляторной активности, строилось три регрессионные модели, в каждой из которых общий уровень осознанной саморегуляции выступал зависимой переменной. Регрессионный анализ проводился по всей выборке испытуемых.

Модель «Мощность» совокупное влияние всех предикторов объясняет 56\% дисперсии. Первым в модель входит фактор мощности актуального образа состояния. Именно его динамика, в большей мере, объясняет изменение процессов управления собой и трудной жизненной ситуацией. Достоверным оказывается и мощность образа состояния при успешном совладании с ситуацией. Индексация мощности по двум данным условиям обусловливает подвижность саморегуляции. С учётом корреляционных значений, увеличение количе- ства характеристик в актуальном состоянии и при успешном опыте разрешения проблем повышает уровень саморегуляции произвольной активности в трудной жизненной ситуации. Сам параметр мощности среди других оказывается наиболее сильным фактором осознанной саморегуляции активности субъекта в трудной жизненной ситуации. Именно от его показателей в большей мере зависит уровень регуляторной активности.

В модели «Структурированность» влияние набора предикторов суммарно объясняет 43\% дисперсии. Надежным фактором во второй модели оказывается индекс структурированности образа состояния в актуальной ситуации. По двум другим условиям дисперсия не превышает 2\%. Другими словами, чем больше структур в своём актуальном состоянии выделяет человек, чем лучше они оформлены, тем эффективнее регуляция произвольной активности. 


\section{Психология и психотехника 10(85) • 2015}

Модель «Интенсивность» (38\% совокупного влияния на зависимую переменную) влияет на осознанную саморегуляцию только посредством образа состояния при успешном совладании, значения которого повышаются после введения в модель двух других индексов. Важно отметить, что индекс интенсивности образа при неуспешном разрешении ситуации, не являясь самостоятельным предиктором, в большей мере обусловливает повышение влияния интенсивности ОУС на осознанную саморегуляцию.

Обобщая результаты проведённого исследования, можно сделать следующие предварительные выводы.

1. Субъективный образ состояния субъекта жизненной ситуации является гибким, подвижным образованием, параметры которого дифференцируются в различных условиях воспроизведения человеком своих характеристик.

2. Формальные параметры субъективного образа состояния в трудной жизненной ситуации меняются в зависимости от выраженности оценки её трудности. Устойчивые параметры мощности, структурированности и интенсивности образа состояния имеют субъекты сла- бо-напряжённой ситуации, позволяющей оптимально адаптироваться к трудным условиям. Интенсивно трудная ситуация, воздействуя на структуры субъективных образов, уменьшает количество и силу характеристик при положительном отражении предыдущего опыта совладания с проблемами и, одновременно, повышает мощность и интенсивность образа при их неуспешном разрешении. Чрезмерно трудная ситуация, не меняя структуры образов, увеличивает их мощность и силу при неуспешном совладания и снижает при успешном.

3. Мощность и интенсивность образов обусловливают зависимость между общей регуляторной активностью и системой субъектных представлений о себе в трудных ситуациях, определяя таким образом степень успешности совладания с проблемами. Прежде всего влияние обусловлено оценкой своих качеств в актуально действующей ситуации.

4. Полученные предварительные данные можно рассматривать как основание для разработки способов коррекционной помощи субъектам трудной жизненной ситуации с учётом воздействия на образ состояния.

\section{Список литературы:}

1. Прохоров А.О. Образ психического состояния: пространственно-временной аспект // Экспериментальная психология в России: традиции и перспективы / Под ред. В.А. Барабанщикова. М.: Институт психологии РАН, 2010. С. 542-547.

2. Дикая Л.Г. Системно-деятельностная концепция саморегуляции психофизиологического состояния человека // Проблемность в профессиональной деятельности: теория и методы психологического анализа. М.: Институт психологии РАН, 1999. С. 80-106.

3. Веккер Л.М. Психика и реальность: единая теория психических процессов. М.: Смысл, 1998. 685 с.

4. Гостев А.А. Психология вторичного образа. М.: Институт психологии РАН, 2007. 512 с.

5. Мартьянова Г.Ю. Особенности регуляторной активности субъекта в трудной жизненной ситуации // Актуальные проблемы психологического знания: теоретические и практические проблемы психологии. 2013. № 3(28). С. 27-39.

6. Моросанова В.И. Личностные аспекты саморегуляции активности человека // Психологический журнал. 2002 . Т. 23. № 6. С. 27-42.

7. Прохоров А.О., Артищева Л.В. Образ психического состояния: динамические и структурные характеристики // Экспериментальная психология. 2012. Т. 5. № 2. С. 63-73.

8. Моросанова В.И. Опросник «Стиль саморегуляции поведения» // Журнал практической психологии и психоанализа. 2004. № 2. С. 24-39.

9. Абульханова К.А. Принцип субъекта в отечественной психологии // Психология. 2005. № 4. С. 3-21.

10. Конопкин О.А. Структурно-функциональный и содержательно-психологический аспекты осознанной саморегуляции // Психология. 2005. № 1. С. 27-42.

11. Варыгин А.Н. «Системное» и «ситуационное» в преступности // Право и политика. 2015. № 1. C. 58-63. (DOI: 10.7256/1811-9018.2015.1.11991).

12. Цветкова М.В. Рефлексивные механизмы субъектных отношений // Политика и общество. 2014. № 4. С. 470-479. (DOI: 10.7256/1812-8696.2014.4.11864).

13. Майданов А.С. Логика мифологического мышления. Часть 2. Парадигмальность мифологического мышления // Философия и культура. 2014. № 4. C. 594-606. (DOI: 10.7256/1999-2793.2014.4.12116).

14. Майданов А.C. Логика мифологического мышления // Философия и культура. 2014. № 3. C. 426-441. (DOI: 10.7256/1999-2793.2014.3.10620). 


\section{References (transliteration):}

1. Prokhorov A.O. Obraz psikhicheskogo sostoyaniya: prostranstvenno-vremennoi aspekt // Eksperimental'naya psikhologiya v Rossii: traditsii i perspektivy / Pod red. V.A. Barabanshchikova. M.: Institut psikhologii RAN, 2010. S. 542-547.

2. Dikaya L.G. Sistemno-deyatel'nostnaya kontseptsiya samoregulyatsii psikhofiziologicheskogo sostoyaniya cheloveka // Problemnost' v professional'noi deyatel'nosti: teoriya i metody psikhologicheskogo analiza. M.: Institut psikhologii RAN, 1999. S. 80-106.

3. Vekker L.M. Psikhika i real'nost': edinaya teoriya psikhicheskikh protsessov. M.: Smysl, 1998. 685 s.

4. Gostev A.A. Psikhologiya vtorichnogo obraza. M.: Institut psikhologii RAN, 2007. 512 s.

5. Mart'yanova G.Yu. Osobennosti regulyatornoi aktivnosti sub"ekta v trudnoi zhiznennoi situatsii // Aktual'nye problemy psikhologicheskogo znaniya: teoreticheskie i prakticheskie problemy psikhologii. 2013. № 3(28). S. 27-39.

6. Morosanova V.I. Lichnostnye aspekty samoregulyatsii aktivnosti cheloveka // Psikhologicheskii zhurnal. 2002 . T. 23. № 6. S. 27-42.

7. Prokhorov A.O., Artishcheva L.V. Obraz psikhicheskogo sostoyaniya: dinamicheskie i strukturnye kharakteristiki // Eksperimental'naya psikhologiya. 2012. T. 5. № 2. S. 63-73.

8. Morosanova V.I. Oprosnik «Stil' samoregulyatsii povedeniya» // Zhurnal prakticheskoi psikhologii i psikhoanaliza. 2004. № 2. S. 24-39.

9. Abul'khanova K.A. Printsip sub"ekta v otechestvennoi psikhologii // Psikhologiya. 2005. № 4. S. 3-21.

10. Konopkin O.A. Strukturno-funktsional'nyi i soderzhatel'no-psikhologicheskii aspekty osoznannoi samoregulyatsii // Psikhologiya. 2005. № 1. S. 27-42.

11. Varygin A.N. «Sistemnoe» i «situatsionnoe» v prestupnosti // Pravo i politika. 2015. № 1. S. 58-63. (DOI: 10.7256/18119018.2015.1.11991).

12. Tsvetkova M.V. Refleksivnye mekhanizmy sub"ektnykh otnoshenii // Politika i obshchestvo. 2014. № 4. S. 470-479. (D0I: 10.7256/1812-8696.2014.4.11864).

13. Maidanov A.S. Logika mifologicheskogo myshleniya. Chast' 2. Paradigmal'nost' mifologicheskogo myshleniya // Filosofiya i kul'tura. 2014. № 4. S. 594-606. (DOI: 10.7256/1999-2793.2014.4.12116).

14. Maidanov A.S. Logika mifologicheskogo myshleniya // Filosofiya i kul'tura. 2014. № 3. S. 426-441. (DOI: 10.7256/19992793.2014.3.10620). 